\title{
Medición de resistencia a tempranas edades del hormigón: método que mejor se ajusta para la determinación de tiempos mínimos de desencofrado de elementos verticales de hormigón
}

\author{
Early age concrete strength measurement: best fit method for the prediction of the minimum \\ vertical concrete elements stripping times \\ Fecha de entrega: 5 de octubre 2016 \\ Fecha de aceptación: 14 de julio 2017
}

\section{Natalia Rudeli y Adrián Santilli}

Departamento de Ingeniería Civil, Universidad de Montevideo, Av. Ing. Luis P. Ponce 1307, Montevideo 11300, Uruguay, nrudeli@correo.um.edu.uy, asantilli@um.edu.uy

En este artículo se presenta un estudio profundo de diferentes métodos para medir resistencia del hormigón a tempranas edades. Se busca determinar el instrumento de medición de resistencia que proporcione el mejor ajuste a las curvas de resistencia - maduración con el fin de determinar tiempos mínimos de desencofrado de elementos verticales de hormigón. Fueron estudiados los ensayos de compresión de probetas cilíndricas de dimensiones normales de $15 x$ $30 \mathrm{~cm}$, compresión de probetas cilíndricas de dimensiones reducidas de $10 \times 20 \mathrm{~cm}$, penetrómetro de hormigón y esclerómetro pendular de baja resistencia. Se utilizaron dos dosificaciones diferentes de hormigón y fueron verificados más de 500 puntos de las curvas. Se concluye que la utilización del esclerómetro pendular es recomendado en caso de que el usuario desee desencofrar a resistencias menores que $3 \mathrm{MPa}$. En caso que se desee desencofrar a resistencias por encima de este valor se recomienda el uso de probetas cilindricas de dimensiones normales.

Palabras claves: hormigón, desencofrado, maduración, coeficientes de maduración, medición de la resistencia a tempranas edades
This article presents a deep study of different methods for early age concrete strength measurement. The goal is the determination of the measuring instrument/ method that best fit the strength-maturity curves in order to determinate minimum vertical concrete stripping times. Compression tests of cylindrical specimens of normal dimensions of $15 \times 30 \mathrm{~cm}$ as well as of smaller dimensions of $10 \times 20 \mathrm{~cm}$ were carried out. In addition, concrete sclerometer tests, low resistance sclerometer and penetrometer test were also studied. Two different concrete dosages were used and the experimental data were checked with more than 500 curves points of the maturity curves. It is concluded that the use of pendular sclerometer is recommended when the user wishes stripping with less than $3 \mathrm{MPa}$ of concrete resistance. If it is desired stripping with resistance above this value, it is recommended the use of cylindrical specimens of normal dimensions.

Keywords: concrete, stripping, maturity, maturity index, early age strength measurement

\section{Introducción}

Durante el proceso constructivo de cualquier obra de hormigón se emplean estructuras temporales denominadas encofrados, las cuales se utilizan para moldear el hormigón fresco a la forma deseada. La utilización de estas estructuras, en general alquiladas, es uno de los costos más importantes en los que incurren las empresas constructoras a la hora de realizar estructuras de hormigón. Hanna y
Senouci (1997) establecen que el costo del encofrado puede ascender hasta el $60 \%$ del costo de una construcción, dato reafirmado recientemente por Kopczynski (2008). Hurd (2005) establece que a la hora de dimensionar un encofrado los tres factores fundamentales son: seguridad, calidad y economía. Por seguridad se entiende que la estructura sea capaz de soportar todos los esfuerzos a los que será 
sometida. Se define calidad como la capacidad del encofrado de proporcionar a la estructura el tamaño, forma, posición y terminación deseada, y por último la economía se refiere al ahorro de tiempo y dinero en el período comprendido entre el llenado y el desencofrado. Si un encofrado vertical es retirado tempranamente, la estructura puede sufrir fallos que afecten a la estabilidad $\mathrm{y} / \mathrm{o}$ funcionalidad de la misma, perjudicando a la seguridad. Por otro lado, una remoción tardía puede originar grandes sobrecostos a las empresas, no sólo por el incremento en el costo del alquiler del encofrado sino porque representa un capital estancado, atrasos en el cronograma de obra y gastos indirectos en este período.

En cuanto a los tiempos mínimos de desencofrado de elementos verticales de hormigón existen diversas normas que predicen su valor. Diferentes autores y normas proponen tiempos mínimos de desencofrado a partir de datos experimentales, pero un estudio realizado por Rudeli et al. (2015) arroja como resultado que existen grandes diferencias entre los valores propuestos para los tiempos mínimos de desencofrado de elementos verticales de hormigón. Por ejemplo, en condiciones de igualación IHA 61 (1961) y Páez (1986) proponen tiempos mínimos de desencofrado de 17 días, mientras que Casinello (1974) y EN 206-1 (2001) proponen 2 días. Para estas mismas condiciones Harmsen (2002), ACI 347 (2004) y BS 8110 (1985) proponen medio día, mientras que Harrison (1977) propone 6 días y medio.

Sumado a esto, hoy en día no existe un método universalmente aceptado para determinar los tiempos mínimos de desencofrado de elementos verticales. En general estos tiempos se determinan en forma arbitraria en obra. Una encuesta llevada adelante en Uruguay a más de 100 personas en el área de la construcción (directores de obra, capataces y oficiales) muestra que el $88 \%$ de los encuestados dicen no tener una regla o método para determinar los tiempos mínimos de desencofrado, mientras que el $70 \%$ de las personas que declaran utilizar un método para determinarlos, dicen que los determinan de modo empírico y según experiencia de obra (Rudeli y Santilli, 2014).

\section{Metodología para desencofrar elementos verticales de hormigón}

La metodología para la determinación de tiempos mínimos presentada por Rudeli et al. (2015) para hormigones tradicionales y reafirmada posteriormente por Santilli et al. (2015) para hormigones autocompactantes se basa en la utilización de coeficientes de maduración. El método de los coeficientes de maduración tiene su origen en estudios realizados por Nurse (1949) y Saul (1951) para métodos de curado acelerado. El método consiste en la determinación de gráficos de maduración para estimar el desarrollo de la resistencia a la compresión y otras propiedades mecánicas del hormigón bajo diferentes condiciones de temperatura. Estos estudios posteriormente fueron estandarizados por la norma ASTM C1074 (2004), la cual recomienda que el coeficiente de maduración sea calculado por medio de:

$$
M=\sum_{0}^{t}\left(T-T_{0}\right) \Delta t
$$

donde $M$ es el valor de maduración a la edad $t$ en ${ }^{\circ} \mathrm{Ch}$, $T$ es la temperatura de curado promedio del hormigón durante el intervalo $\Delta t$ en ${ }^{\circ} \mathrm{C}, T_{0}$ es una temperatura de referencia, $t$ es el tiempo en h (horas) y $\Delta t$ es el periodo de tiempo a la temperatura $T$ en h. Según Brooks et al. (2008), $T_{0}$ es la temperatura a la cual el cemento deja de reaccionar o la temperatura a la cual el hormigón no adquiere resistencia. Tanto Brooks et al. (2008) como Carino et al. (1983) consideran un valor de $T_{0}$ igual a $-10^{\circ} \mathrm{C}$. Se puede decir entonces que para una misma dosificación y el mismo coeficiente de maduración, se tendrá la misma resistencia sin importar la combinación de temperatura y tiempo con la que se obtenga. Es decir que para una dosificación dada la curva resistencia - maduración del hormigón es única. Por lo tanto, puede ser considerada un dato del proyecto, ver Figura 1.La metodología propuesta por Rudeli et al. (2015) consiste en construir la curva resistencia - maduración mostrada en la Figura 1, mediante ensayos de probetas cilíndricas (ASTM C1074, 2004) y utilizando la expresión (1). Posteriormente, conociendo la resistencia a la que se 


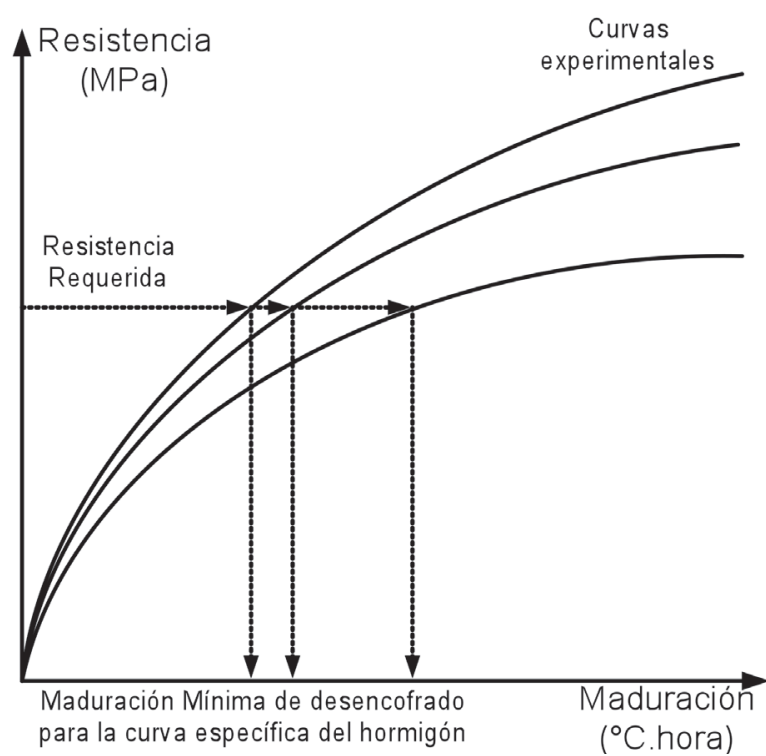

Figura 1: Curva resistencia - maduración

desea desencofrar se determina la maduración mínima para el desencofrado. Por lo que basta con seleccionar la resistencia que se desea alcanzar a la hora de desencofrar para determinar por medio de la curva mencionada la maduración correspondiente. De este modo se puede retirar el encofrado una vez que el hormigón haya alcanzado la resistencia requerida por proyecto. Puede decirse entonces que la mayor limitación de la metodología para determinar tiempos mínimos de desencofrado viene dada por la medición de resistencia in situ a tempranas edades del hormigón. Es por esto que en este artículo se plantea un estudio profundo de la metodología más adecuada para la medición de resistencia a tempranas edades del hormigón, enfocado en la determinación de los tiempos mínimos de desencofrado a tempranas edades.

\section{Métodos para medir resistencia del hormigón}

En este apartado se describirán los métodos conocidos en la literatura para la medición de resistencia del hormigón a temprana edad. De este modo mediante una comparativa técnica se podrá decidir los mecanismos para la medición de resistencia del hormigón más convenientes para la realización de la campaña experimental.

\section{Rotura a compresión de especímenes}

Existen tres tipos de probetas que en general se utilizan para determinar la resistencia a la compresión: probetas cilíndricas de $15 \times 30 \mathrm{~cm}$, probetas cilíndricas de 10 x $20 \mathrm{~cm}$ y probetas cúbicas. En todos los casos el objetivo principal del ensayo es determinar la máxima resistencia a la compresión de un espécimen frente a una carga aplicada axialmente. Es un ensayo universalmente aceptado para ejecutar las pruebas de resistencia mecánica de compresión simple y se encuentra descrito por la EHE 08 (2008), UNE EN 12390 (2003) y NTC 673 (2010), entre otras.

\section{Pull-out o Lok-test}

El ensayo pull-out o Lok-test, está especialmente diseñado para medir la resistencia del hormigón y consiste en medir el máximo esfuerzo necesario para extraer una pieza metálica embebida en una masa de este material. El procedimiento se encuentra normalizado según la norma ASTM C900 (2013). Una de las principales desventajas de este ensayo es que se debe tomar en cuenta la variación de resistencia del hormigón a lo largo de la estructura. Adicionalmente, este ensayo es poco utilizado ya que requiere mano de obra capacitada y buena planificación de los puntos de ensayo (Germann Instruments, germann.org).

\section{Break-off test}

El ensayo conocido como break-off o de fractura interna, es utilizado frecuentemente para la determinación de la calidad de las estructuras. Consiste en determinar la carga que provoca la flexión de un cilindro de $55 \mathrm{~mm}$ de diámetro por $70 \mathrm{~mm}$ de longitud ubicado en el hormigón in situ. El ensayo es descrito por Di Maio et al. (1996). Según Bazant y Planas (1998) la limitación más importante de este procedimiento es que las curvas de correlación que se obtienen mediante ensayos empíricos dependen de varios factores como por ejemplo las condiciones de curado del hormigón. Por lo que la relación entre el resultado del ensayo y la estimación de las propiedades del material son estimaciones que conllevan grandes variaciones.

\section{Ultrasonido}

El método ultrasónico se basa en un fenómeno físico conocido como propagación de ondas en un medio 
material, este método se utiliza frecuentemente cuando se desea determinar la uniformidad de un elemento, su espesor o módulo elástico. Sin embargo, el método tiene grandes limitantes cuando el material es heterogéneo ya que las diferentes fases que lo componen tienen diferentes propiedades elásticas relativas a la propagación de las ondas. Este método tiene como principal ventaja el no necesitar una única medida para obtener un valor representativo, es decir, que la dispersión del ensayo es baja (del orden del $2.5 \%$ ). Este método de control del hormigón con emisión de impulsos generados mecánicamente se basa en que la velocidad de onda a través del hormigón depende de las propiedades elásticas y es casi independiente de la geometría estudiada (UNE 83308, 1986). A pesar de la utilidad de este ensayo, se recomienda la utilización del mismo a partir de los 15 días posteriores del llenado dado que la influencia del contenido de agua inicial puede hacer variar las mediciones. Es por ello que este no se considera un método adecuado para la medición de resistencia a tempranas edades.

\section{Esclerómetro de alta y baja resistencia}

El esclerómetro es una herramienta para medir la resistencia a la compresión del hormigón endurecido. Consiste en soltar un martillo metálico que está tensado con un resorte contra la superficie del hormigón, y medir la distancia de rebote. El martillo golpea a la superficie por medio de un percutor y el aparato arroja un número de rebote que puede ser relacionado con la resistencia a la compresión del hormigón. Según la norma ASTM C805/C805M (2013), esta relación depende de la dosificación del hormigón y del aparato utilizado. Existen dos tipos de esclerómetros: de alta resistencia o de baja resistencia. El esclerómetro de alta resistencia es el más utilizado para la medición de resistencias in situ de hormigones de avanzadas edades y comprobaciones de estados edilicios entre otros. ASTM C805/C805M (2013) menciona que cuando el martillo es arrojado, con una determinada energía inicial, e impacta contra la superficie del hormigón, se produce una redistribución de esta energía: una parte es absorbida como fricción dentro del aparato, otra es liberada como energía de deformación del hormigón y la restante se convierte en energía cinética del martillo. Por lo tanto la distancia de rebote del martillo es una medida de la deformación del hormigón, ya que depende de esta energía de movimiento restituida, la cual está relacionada con la deformación del mismo (y así de su resistencia superficial). Según la misma norma, este tipo de ensayos comienza a arrojar resultados con baja incertidumbre a partir de 15 a 16 días de endurecido el hormigón, es por ello que el martillo de alta resistencia no puede ser utilizado para medir resistencias iniciales del hormigón durante la campaña experimental.

Por otro lado los esclerómetros de baja resistencia o martillos pendulares de Schmidt han sido concebidos para ejecutar ensayos en materiales más blandos que el hormigón endurecido, como puede ser paneles de yeso, hormigón fresco y mortero en las juntas de unión de mampostería. Este martillo mide el rebote pendular y por lo tanto requiere ser cargado al igual que el anterior sobre la superficie. La superficie más grande del punzón permite ensayos de hormigón blando a partir de una resistencia a la compresión de $1 \mathrm{MPa}$ y será ensayado durante la campaña experimental.

\section{Sonda de Windsord}

El sistema de sondas HP Windsor ${ }^{\circledR}$ se utiliza para evaluar la resistencia a la compresión del hormigón. El método general de ensayo se lleva a cabo mediante la penetración de la superficie del hormigón con una sonda de acero endurecido con una punta cónica. La prueba de Windsor $^{\circledR}$ ha sido descrita por las normas ASTM C803/C803M (2003). En cuanto a las limitaciones de este método Arni (1973) y Malhorta (1970) indican que los fabricantes no siempre proveen tablas con resultados satisfactorios y debido a esto el ensayo no fue utilizado durante la campaña experimental.

\section{Penetrómetro}

La norma ASTM C403/403M (2008) permite determinar la resistencia a la penetración y por lo tanto determinar los efectos del tiempo de fraguado sobre las características de endurecimiento del hormigón. Para 
este ensayo se construye un testigo del mortero a medir con aproximadamente $9.5 \mathrm{~cm}$ de altura (en un recinto indeformable) y se realizan mediciones continuas en series de 3 (para tomar promedios ente las mismas) cada intervalos de tiempos regulares con el fin de construir una curva de resistencia a la penetración en función del tiempo. A medida que las agujas de mayor diámetro no pueden penetrar en el hormigón se desciende la superficie de contacto y se cambia por una aguja de menor espesor, hasta el punto de no penetración. Este es un ensayo relativamente fácil de realizar y proporciona una idea de endurecimiento del hormigón, es por ello que será utilizado durante el transcurso de la campaña experimental.

\section{Campaña experimental}

La campaña experimental llevada a cabo durante esta investigación se basa en la determinación del mejor método para medir resistencia. Se entiende el mejor método como aquel que se adapta mejor a la metodología presentada por Rudeli et al. (2015) y que por lo tanto permite predecir con menor error la resistencia del hormigón a tempranas edades y determinar con mayor precisión el tiempo mínimo de desencofrado de elementos verticales de hormigón. Se busca principalmente el método para medir resistencia que genere menores dispersiones en la predicción de resistencias a tempranas edades del hormigón. Es por ello que se diseñó una serie de ensayos abocados a la determinación de la resistencia a tempranas edades utilizando varios de los métodos ya descritos.

\section{Materiales y procedimientos}

\section{Métodos para medir resistencia}

Durante el transcurso de la campaña experimental se utilizaron 4 metodologías para medir resistencia a tempranas edades: probetas cilíndricas de dimensiones normales, probetas cilíndricas de dimensiones reducidas, esclerómetro de baja resistencia y penetrómetro.

a) La medición de la resistencia a la compresión mediante la utilización de especímenes cilíndricos de $30 \times 15 \mathrm{~cm}$ (conocido como de dimensiones normales) es recomendada por la normativa ASTM C1074 (2004), pero atendiendo al aumento teórico de resistencia que se obtiene mediante la utilización de probetas de menores dimensiones, esto debido al mayor confinamiento que sufren las probetas durante la ejecución del ensayo y que tiene como resultado un aumento de la resistencia, en esta campaña experimental se utilizan también probetas cilíndricas de dimensiones reducidas de 10 x $20 \mathrm{~cm}$.

b) A pesar que el uso del esclerómetro de alta resistencia no está recomendado para mediciones a tempranas edades (ASTM C805/C805M, 2013), el uso de esclerómetros de baja resistencia o esclerómetro de péndulo para la medición indirecta de resistencia a la compresión es altamente recomendado y será testeado junto a los demás métodos para medir resistencia a tempranas edades.

c) El ensayo por penetración permite tener una idea del proceso que vive el hormigón mientras endurece y que su utilización también es recomendable.

Durante el transcurso de la campaña experimental se comparará el desempeño de cada uno de estos instrumentos y/o métodos de medición de resistencias a tempranas edades del hormigón para determinar el más apropiado para ser utilizado en referencia al método de coeficientes de maduración.

\section{Método para medir madurez}

Para la obtención de los valores de maduración, durante este programa experimental se utilizó un cable de termopar tipo K como se muestra en la Figura 2. Los datos de temperatura se registraron en intervalos de tiempo iguales de 5 min y fueron transformados a valores de maduración utilizando (1) y considerando $T_{0}=0$ según ASTM C1074 (2004).

\section{Materiales}

Para este programa experimental se seleccionaron dos dosificaciones D1 y D2 que se muestran en la Tabla 1. El cemento utilizado es cemento Portland de resistencia normal de $40 \mathrm{MPa}$ a los 28 días (CEMI 42.5 N según EN 197-1, 2006). El módulo granulométrico del agregado grueso (compuesta por los agregados de 
$3 \mathrm{~mm}<\Phi \leq 12 \mathrm{~mm}$ y $0.425 \mathrm{~mm}<\Phi \leq 4.75 \mathrm{~mm})$ es 5.54, mientras que el módulo granulométrico del agregado fino es 2.90 .

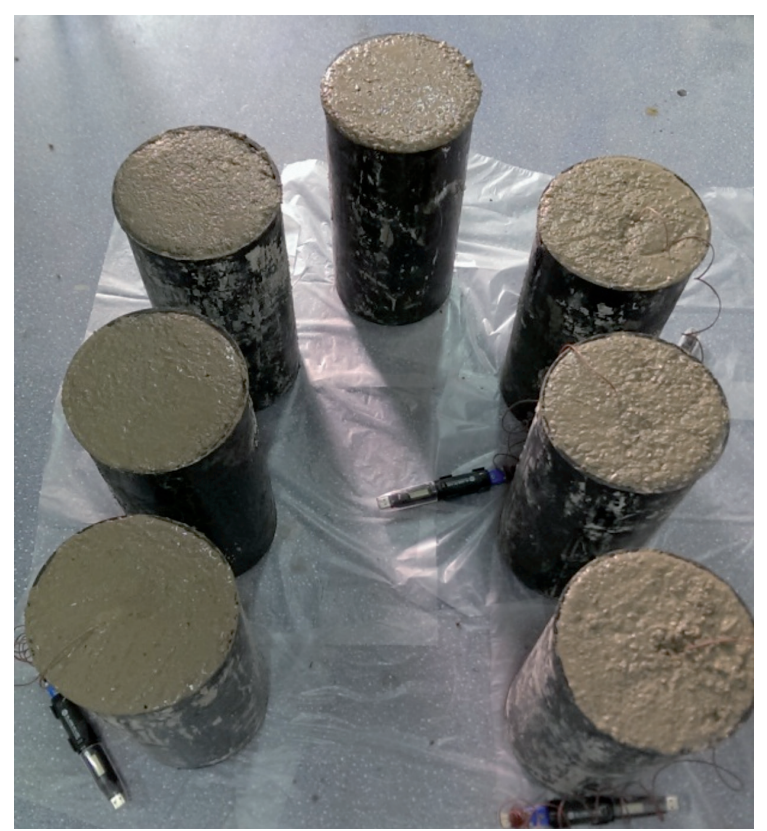

Figura 2: Probetas cilíndricas de dimensiones normales con cables termopares

Tabla 1: Dosificaciones de hormigón para el programa experimental

\begin{tabular}{|l|c|c|}
\hline Componente & $\begin{array}{c}\text { Peso por } \\
1000 \mathrm{~kg}(\mathrm{D} 1)\end{array}$ & $\begin{array}{c}\text { Peso por } \\
1000 \mathrm{~kg}(\mathrm{D} 2)\end{array}$ \\
\hline $\begin{array}{l}\text { Agregado grueso, } \\
3<\Phi \leq 12 \mathrm{~mm}\end{array}$ & 220.5 & 209.6 \\
\hline $\begin{array}{l}\text { Agregado grueso, } \\
0.425<\Phi \leq 4.75 \\
\mathrm{~mm}\end{array}$ & 220.5 & 209.6 \\
\hline $\begin{array}{l}\text { Agregado fino, } \\
\Phi \leq 3 \mathrm{~mm}\end{array}$ & 336.0 & 331.1 \\
\hline $\begin{array}{l}\text { CPN40 cemento } \\
\text { Portland }\end{array}$ & 146.7 & 134.8 \\
\hline Agua & 76.2 & 114.7 \\
\hline
\end{tabular}

\section{Procedimientos}

Este programa experimental tiene como objetivo principal la determinación de la mejor metodología para la medición de resistencias a tempranas edades. La norma ASTM C1074 (2004) recomienda la utilización de probetas cilíndricas para la construcción de la curva resistencia - maduración. A pesar de ello, los rangos de resistencias con las que se trabaja en esta curva son mayores a los que Rudeli et al. (2015) recomiendan para el desencofrado de elementos verticales de hormigón. Es por ello que en esta sección se compararán varios métodos para determinar cual es más eficiente a la hora de construir la curva resistencia - maduración, trabajando a tempranas edades de desencofrado. Para cada método seleccionado fueron obtenidas las curvas de resistencia-maduración mediante la medición en intervalos regulares de resistencia. Posteriormente, siguiendo el mismo procedimiento utilizado para la construcción de las curvas de caracterización, para cada una de las dosificaciones y métodos de medir resistencia se determinaron puntos adicionales, al menos 15 puntos adicionales para cada una de las mezclas y métodos de medir resistencia. Estos puntos adicionales fueron utilizados para establecer la precisión con la cual la curva de caracterización predice resistencias a tempranas edades. Para la maduración de cada punto de verificación, fue obtenida la resistencia teórica (utilizando la curva de caracterización de la mezcla) y esta resistencia teórica fue comparada con la resistencia real obtenida.

Posteriormente se determinó la desviación estándar de los puntos de verificación de la curva de caracterización. De este modo la metodología para medir resistencia que tenga menor desviación de la curva de caracterización será la metodología que proporciona menor error a la hora de medir resistencia y por lo tanto la recomendada para la medición de la misma a tempranas edades.

\section{Resultados y análisis}

Las curvas de caracterización para ambas dosificaciones y los cuatro métodos para medir resistencia se muestran en las Figuras 3 y 4 . En el caso de penetrómetro y esclerómetro, debido a la gran dispersión de los valores a edades tardías se presentan dos comparativas de los valores experimentales: uno que incluye la curva completa y otro en la que se tiene en cuenta únicamente el tramo inicial de la curva, en donde las dispersiones de los resultados son menores. Se obtiene entonces dos dispersiones, una para el ajuste de la curva cuando se trabaja a edades bajas del hormigón y otro ajuste de la curva cuando se trabaja con la curva completa. 


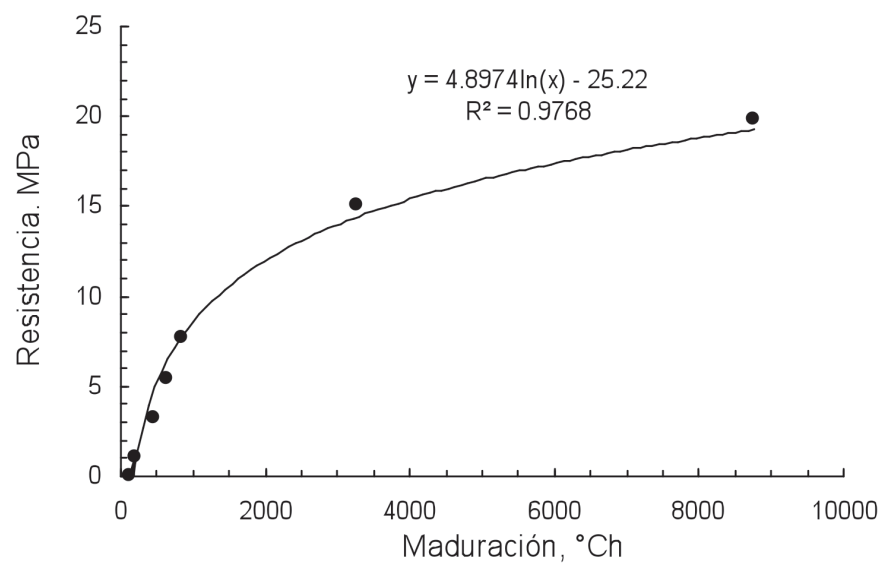

(a) $\mathrm{D} 1,15 \times 30 \mathrm{~cm}$

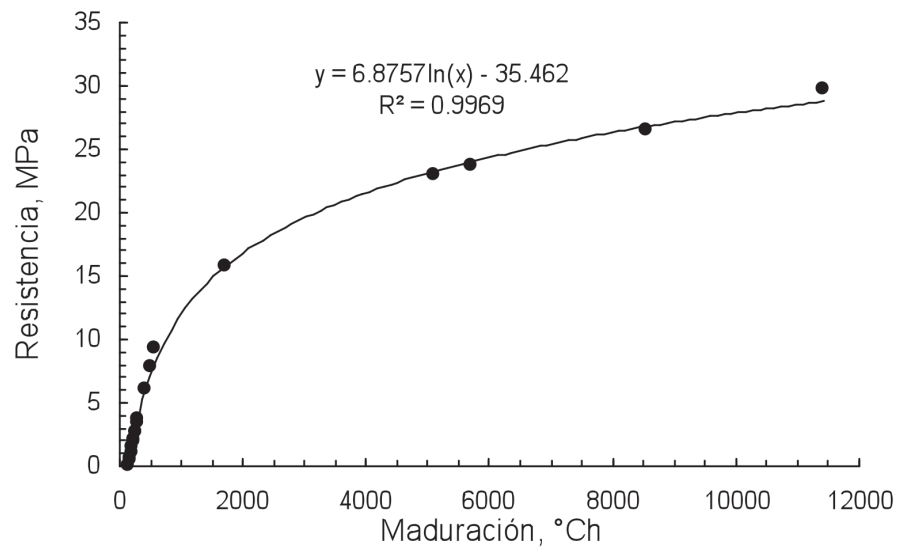

(b) $\mathrm{D} 2,15 \times 30 \mathrm{~cm}$

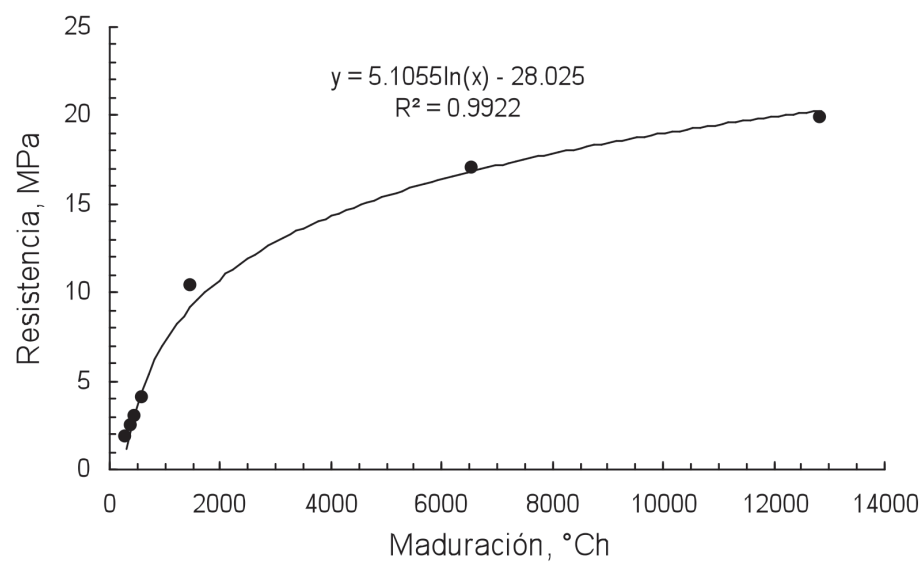

(c) D1, $10 \times 20 \mathrm{~cm}$

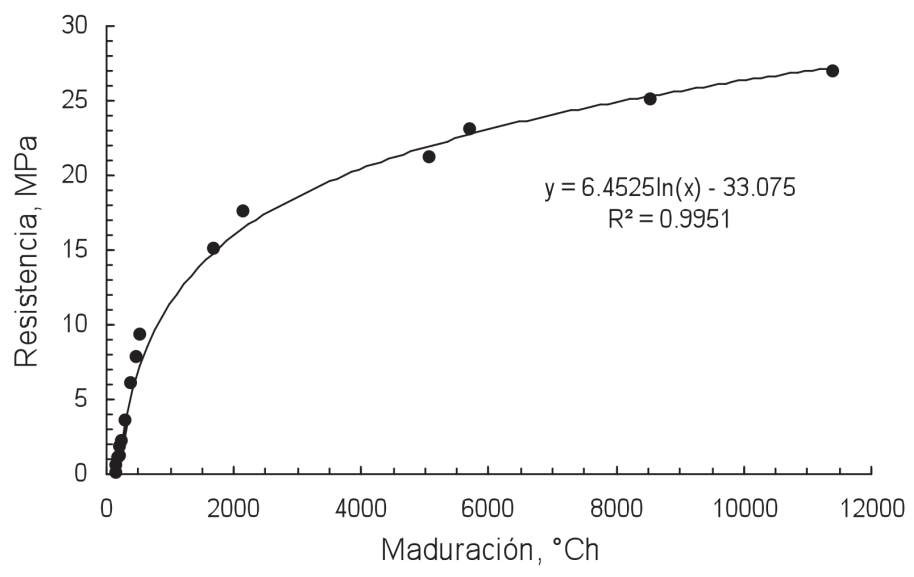

(d) $\mathrm{D} 2,10 \times 20 \mathrm{~cm}$

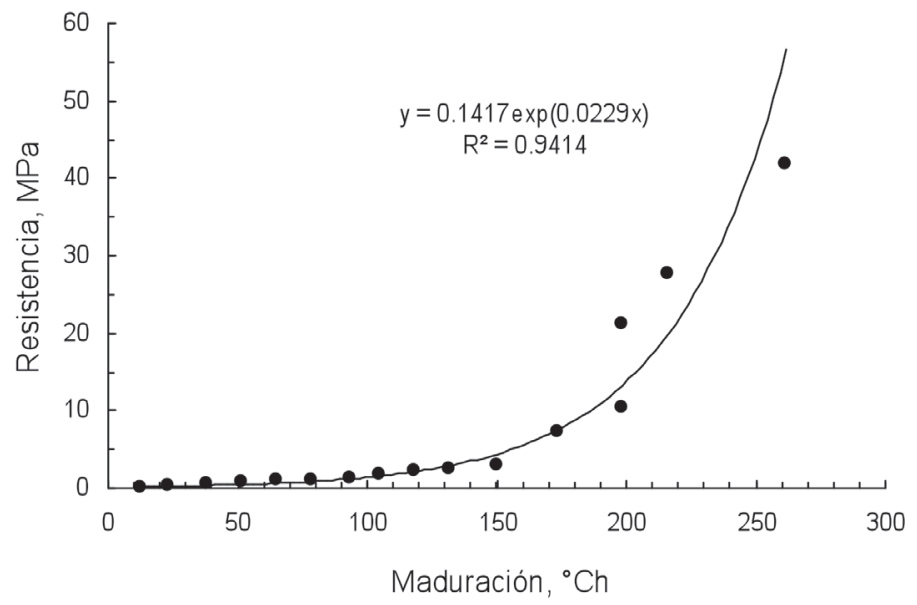

(e) Curva completa D1, penetrómetro

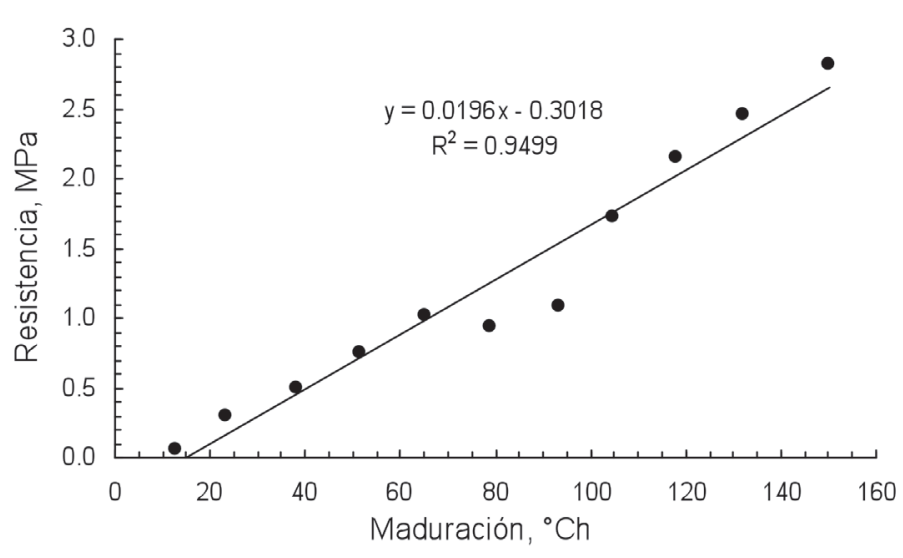

(f) Curva completa D1, esclerómetro

Figura 3: Curvas de caracterización 
Esta resistencia teórica fue comparada con la resistencia real obtenida mediante la nueva medición y determinado el error con el cual la curva de caracterización original predijo la resistencia que se midió a cada maduración. Por ejemplo, para una probeta cilíndrica de dimensiones normales trabajando con la dosificación D1, la curva de caracterización viene dada por la ecuación: Resistencia $=4.8974$ $\ln$ (Maduración)-25.22 (Figura 3a).

Uno de los valores de verificación obtenidos para una probeta cilíndrica de dimensiones normales es de 7.96 MPa a una maduración de $859^{\circ} \mathrm{Ch}$. La maduración de este nuevo punto fue tomada y determinada entonces la resistencia teórica que la curva de caracterización predice, en este caso la resistencia teórica es de 7.87 MPa. Fue entonces obtenido el valor de desviación estándar y el error de la medición mediante la utilización de la siguiente expresión:

Error $(\%)=\frac{\text { Resistencia teórica }- \text { Resistencia real }}{\text { Resistencia real }} \times 100$

En este caso la desviación del valor puntual es de 0.11 $\mathrm{MPa}^{2}$ y el error porcentual es de $4.15 \%$. El promedio de las desviaciones de todos los valores es considerado la desviación estándar de la metodología de la curva de caracterización y da una idea del ajuste de los valores experimentales a la curva. Adicionalmente fue determinado el error porcentual expresado según (2).

Esta metodología fue repetida para cada una de las dosificaciones y para cada uno de los métodos para medir resistencia a tempranas edades. En la Tabla 2 se presenta un resumen de los valores obtenidos. Se puede concluir que en cuanto a las probetas cilíndricas, las probetas de dimensiones reducidas presentan el doble de dispersión que las probetas de dimensiones normales. Es por ello que si se ha de optar por la medición de resistencia mediante compresión de testigos cilíndricos el método más confiable, en lo que se refiere a la metodología para determinar tiempos mínimos de desencofrado, es mediante probetas de dimensiones normales.
Tabla 2: Resumen de dispersiones de metodologías para medir resistencia

\begin{tabular}{|l|c|c|c|c|c|c|}
\hline \multirow{2}{*}{} & \multicolumn{2}{|c|}{$\begin{array}{c}\text { Desviación, } \\
\mathrm{MPa}^{2}\end{array}$} & \multicolumn{2}{c|}{ Error, \% } & \multicolumn{2}{c|}{$\mathrm{N}^{\circ}$ de datos } \\
\cline { 2 - 7 } & $\mathrm{D} 1$ & $\mathrm{D} 2$ & $\mathrm{D} 1$ & $\mathrm{D} 2$ & $\mathrm{D} 1$ & $\mathrm{D} 2$ \\
\hline $\begin{array}{l}\text { Probetas de } \\
\text { dimensiones } \\
\text { normales }\end{array}$ & 0.33 & 0.29 & 14.3 & 12.2 & 19 & 14 \\
\hline $\begin{array}{l}\text { Probetas de } \\
\text { dimensiones } \\
\text { reducidas }\end{array}$ & 0.65 & 0.62 & 30.6 & 33.2 & 17 & 10 \\
\hline $\begin{array}{l}\text { Penetrómetro } \\
\text { con curva } \\
\text { completa }\end{array}$ & 40 & 41 & 68.7 & 73.9 & 42 & 45 \\
\hline $\begin{array}{l}\text { Penetrómetro } \\
\text { hasta 3 MPa }\end{array}$ & 0.24 & 0.21 & 20.3 & 19.4 & 28 & 32 \\
\hline $\begin{array}{l}\text { Esclerómetro } \\
\text { con curva } \\
\text { completa }\end{array}$ & 0.64 & 0.65 & 45.0 & 38.9 & 85 & 87 \\
\hline $\begin{array}{l}\text { Esclerómetro } \\
\text { hasta 3 MPa }\end{array}$ & 0.11 & 0.10 & 9.1 & 8.3 & 76 & 72 \\
\hline
\end{tabular}

En lo que se refiere al uso de penetrómetro, claramente hay dos zonas definidas: la porción inicial en la que se destaca una menor dispersión, y el uso de la curva completa hasta el punto de no penetración, la cual proporciona una dispersión mucho mayor. En el caso del esclerómetro se produce una situación similar en donde la utilización de la curva completa arroja desviaciones excesivas a edades tardías. En la porción inicial de la curva (hasta $3 \mathrm{MPa}$ ) existe menor dispersión de los resultados, pero a partir de $3 \mathrm{MPa}$ las desviaciones que se obtienen a través del uso de esclerómetro de baja resistencia comienzan a ser significativas.

En base a los resultados obtenidos, se puede afirmar que el uso del esclerómetro hasta $3 \mathrm{MPa}$ presenta menores dispersiones que las otras metodologías para medir resistencia. De hecho, presenta en promedio 3 veces menos desviación que las probetas cilíndricas de dimensiones normales y 2 veces menos dispersión que el uso del penetrómetro. Teniendo esto en cuenta y las resistencias mínimas de desencofrado que proponen las normas y autores (2 MPa la menor de ellas), se puede decir que es recomendado entonces el uso del esclerómetro de baja resistencia, pero si el usuario de la metodología desea desencofrar a resistencias mayores a $3 \mathrm{MPa}$ se recomienda entonces el uso de probetas cilíndricas de dimensiones normales. 


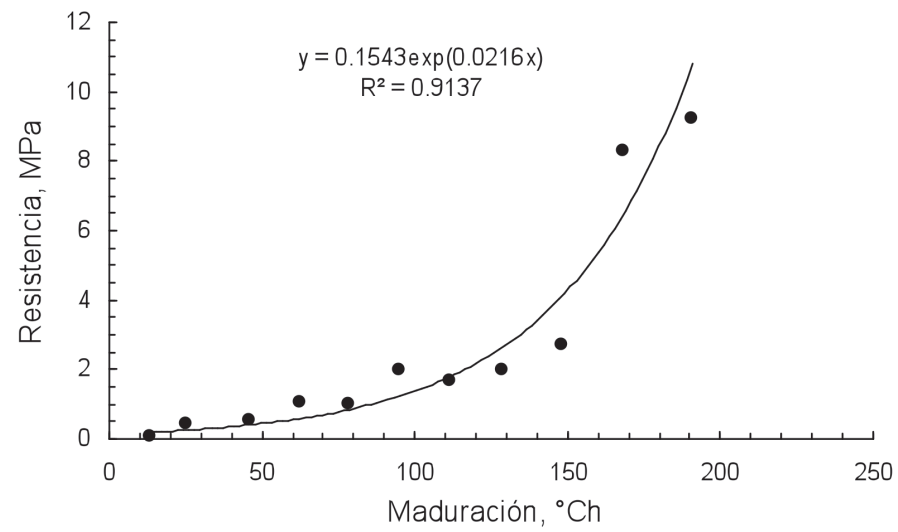

(a) Curva completa D2, penetrómetro

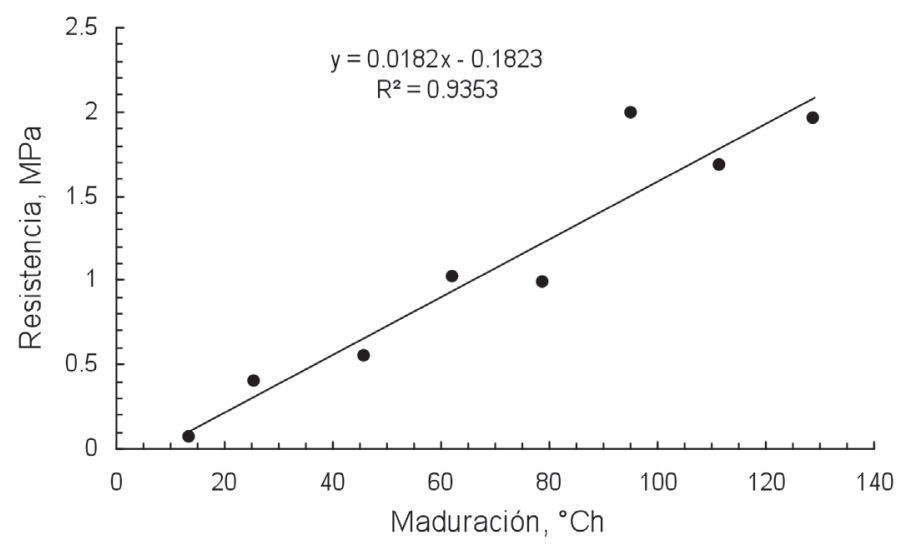

(b) Curva inicial D2, penetrómetro

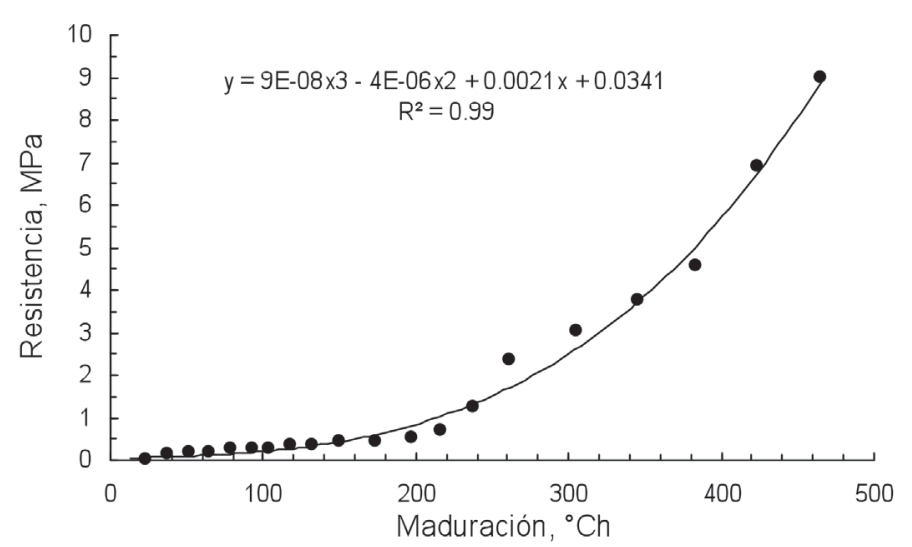

(c) Curva completa D1, esclerómetro

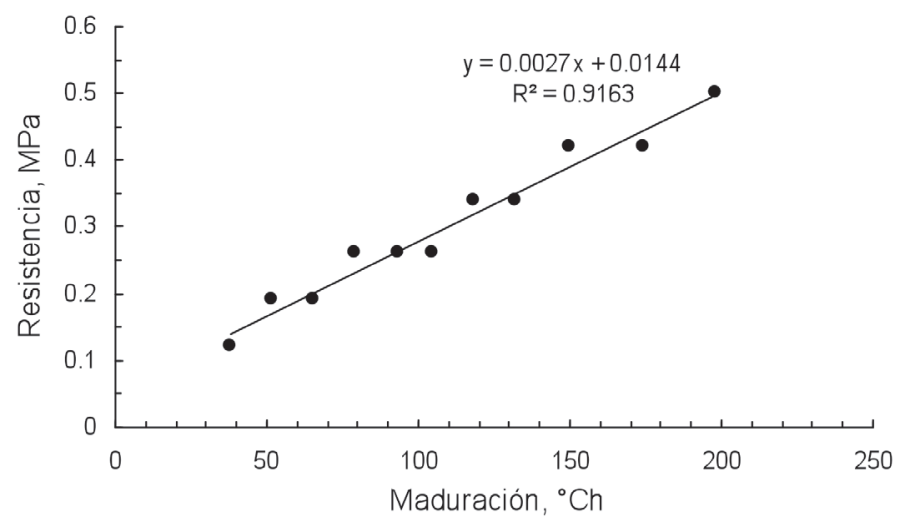

(d) Curva inicial D1, esclerómetro

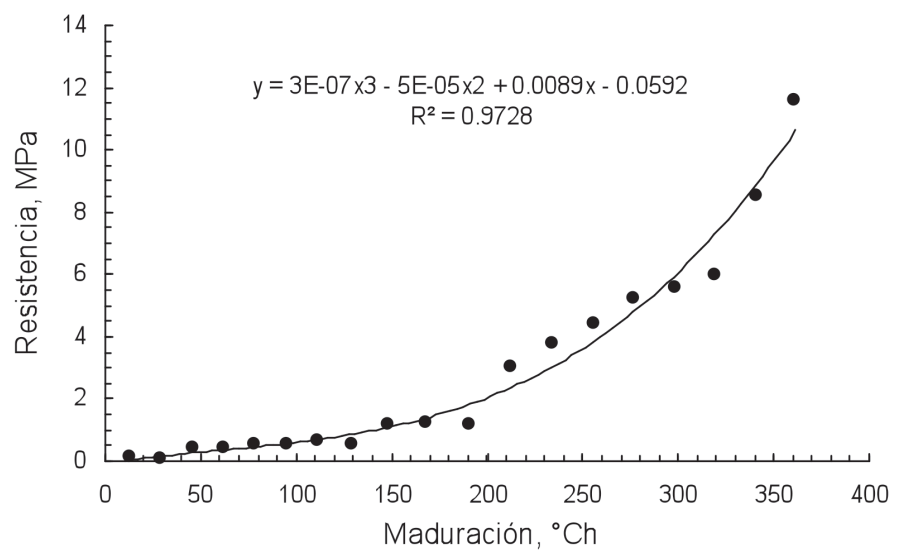

(e) Curva completa D2, esclerómetro

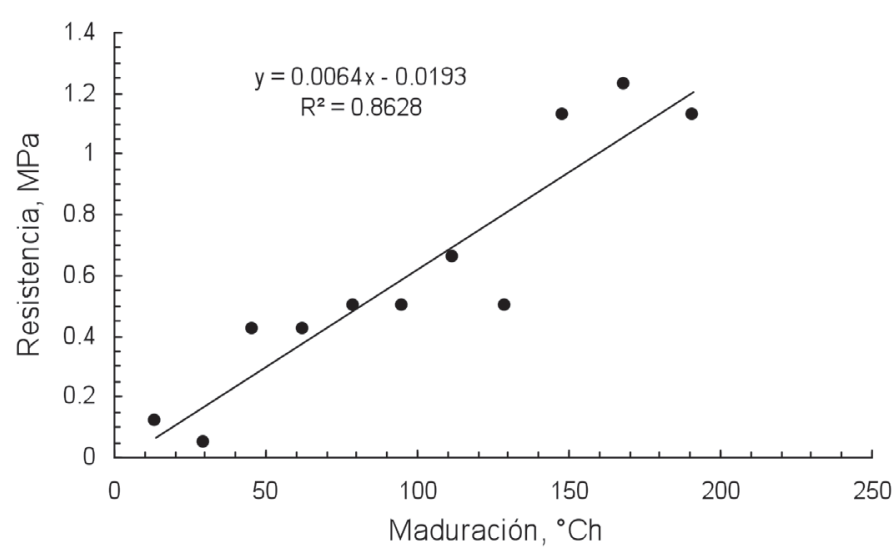

(f) Curva inicial D2, esclerómetro

Figura 4: Curvas de caracterización 


\section{Conclusión}

En este artículo se busca determinar el mejor instrumento para medir resistencias a tempanas edades que se ajuste a la metodología para determinar tiempos mínimos de desencofrado de elementos verticales de hormigón. Luego de un estudio realizado para dos dosificaciones diferentes de hormigón vibrado, para cuatro métodos de medición de resistencia y verificaciones llevadas a cabo en más de 500 puntos, se puede concluir que si el usuario de la metodología para determinar tiempos mínimos de desencofrado desea desencofrar a resistencias menores que $3 \mathrm{MPa}$ entonces es recomendado el uso de esclerómetro de baja resistencia para la construcción de las curvas de caracterización, mientras que si se desea desencofrar a resistencias mayores por razones de seguridad $u$ otras que pudiesen tener que ver con el proyecto, es recomendable la utilización de probetas cilíndricas de dimensiones normales de 15 x $30 \mathrm{~cm}$.

\section{Referencias}

ACI 347 (2004). Guide to formwork for concrete. American Concrete Institute, Farmington Hills, USA

Arni, H.T. (1972). Impact and penetration tests of portland cement concrete. Federal Highway Administration Report No FHWA-RD-73-5.

ASTM C403/C 403M (2008). Standard test method for time of setting of concrete mixtures by penetration resistance. American Society for Testing and Materials. West Conshohocken, USA

ASTM C803/C803M (2003). Standard test method for penetration resistance of hardened concrete. American Society for Testing and Materials. West Conshohocken, USA

ASTM C805/C805M (2013). Standard test method for rebound number of hardened concrete. American Society for Testing and Materials. West Conshohocken, USA

ASTM C900 (2013). Standard test method for pullout strenght of hardened concrete. American Society for Testing and Materials. West Conshohocken, USA

ASTM C1074 (2004). Standard practice for estimating concrete strength by the maturity method. American Society for Testing and Materials. West Conshohocken, USA

Bazant, Z.P. and Planas, J. (1998). Fracture and size effect in concrete and other quasibrittle materiales. CRC press

Brooks, A., Schindler, A. and Barnes, R. (2008). Maturity method evaluated for various cementitious materials. Journal of Materials in Civil Engineering 19, 1017-1025

BS 8110 (1985). Structural use of concrete. Part 2: Code of practice for special circumstances. British Standards Institution, Milton Keynes, UK

Carino, N.J., Lew, H.S. and Volz, C.K. (1983). Early age temperature effects on concrete strength prediction by the maturity method. Journal of the American Concrete Institute 80(2), 93-101

Casinello, F. (1974). Construcción: hormigonería. Rueda, Madrid

Di Maio, A., Giaccio, G. and Zerbino, R. (1996). Break-off test for higth-strength concrete. Cement Concrete and Aggregate 18(1), 15-18

EHE-08 (2008). Instrucción de hormigón estructural. Gobierno de España, Ministerio de Fomento

EN 206-1 (2001). Concrete - Part 1: Specification, performance, production and conformity. Concrete and related products technical committee. European Standard

EN 197-1 (2006). Composition, specifications and conformity criteria for common cements. European Standard

Hanna, A.S. and Senouci,A.B. (1997). Material cost minimization of concrete wall forms. Building and Environment 32(1), 57 - 67

Harrison, T. (1977). Tables of minimum striking times for soffit and vertical formwork. CIRIA Report 67

Harmsen, T. (2002). Diseño de estructuras de concreto armado. Fondo Editorial PUC del Perú, tercera edición

Hurd, M.K. (2005). Formwork for concrete. American Concrete Institute, Farmington Hills

IHA 61 (1961). Instrucción H.A. 61. Especial para estructuras de hormigón armado. Normas y manuales del Instituto Eduardo Torroja de la construcción y del cemento, Madrid

Kopczynski, C. (2008). Formwork efficiencies. Concrete International 30(6), $41-43$ 
Malhorta, V.M. (1970). Preliminary evaluation of Windsor probe equipment for estimating the compressive strength of concrete. Mines Branch Investigation Report IR 71-1, Department of Energy, Mines and Resources, Ottawa

NTC 673 (2010). Concretos: ensayo de resistencia a la compresión de especímenes cilíndricos de concreto. Norma Técnica Colombiana.

Nurse, R.W. (1949). Stream curing of concrete. Magazine of Concrete Research 1(2), 79-88

Páez, A. (1986). Hormigón Armado. Editorial Reverte

Rudeli, N., Santilli, A. and Arrambide, F. (2015). Striking of vertical concrete elements: An analysis using the maturity method. Engineering Structures 95, 40-48

Rudeli, N. y Santilli, A. (2014). Tiempos de desencofrado de elementos verticales de hormigón armado: Método a través de coeficientes de maduración y encuesta en Uruguay. Memorias de Trabajos de Difusión Científica y Técnica.
Santilli, A., Texeira, S. and Puente, I. (2015). Influence of temperature and concrete reinforcement on vertical formwork design. Construction and Building Materials 88, 188-195

Saul, A.G. (1951). Principles underlyng the steam curing of concrete at atmospheric pressure. Magazine of Concrete Research 2(6), 127-140

UNE EN 12390 (2003). Ensayos de hormigón endurecido. Parte 3: Determinación de la resistencia a compresión de probetas. AENOR España

UNE 83308 (1986). Ensayos de hormigón: determinación de la velocidad de propagación de los impulsos ultrasónicos. AENOR España 